\title{
Beneficial Effects of Human Papillomavirus Vaccine for Prevention of Cervical Abnormalities in Miyagi, Japan
}

\author{
Nobuyoshi Ozawa, ${ }^{1}$ Kiyoshi Ito, ${ }^{2}$ Toru Tase,${ }^{3}$ Hirohito Metoki ${ }^{4}$ and \\ Nobuo Yaegashi ${ }^{5}$

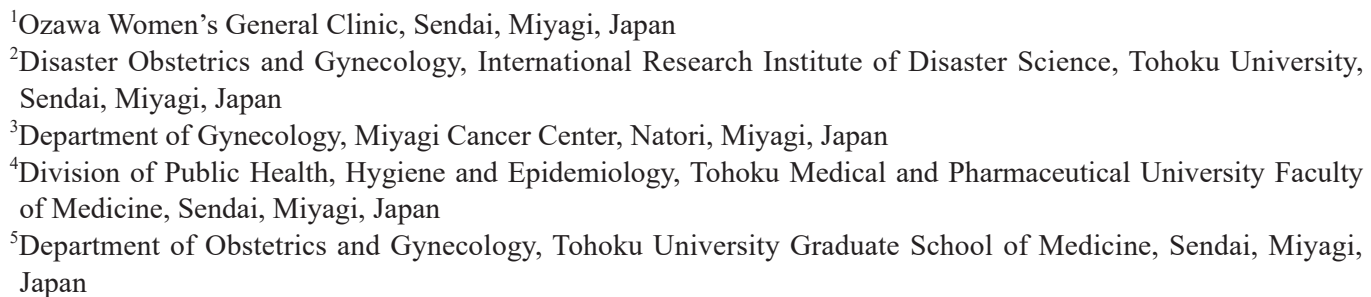

Prevention of cervical cancer has been unsuccessful in Japan because of low rates of cancer screening and vaccination. The Vaccine Adverse Review Committee of the Japanese Government investigated 2,475 adverse events and reported $617(6.9 / 100,000)$ severe cases and $176(2.0 / 100,000)$ cases with chronic pain. The proactive recommendation for human papillomavirus (HPV) vaccination has been suspended since June 2013. In this study, we examined vaccination rate and incidence of abnormal cervical cytology in women aged 20 to 24 years attending cancer screening in Miyagi. Among the 3,272 women who underwent a health check in the fiscal year 2014 (April 2014-March 2015), 332 (10.2\%) received a HPV vaccination. The HPV vaccination rates were $42.3 \%, 10 \%, 17.5 \%, 3.8 \%$ and $4.0 \%$ in women aged 20,21 , 22, 23 and 24 years, respectively. The rates of atypical squamous cells of undetermined significance (ASC-US) or worse were $2.41 \%(8 / 332)$ in women with HPV vaccination and $5.03 \%(148 / 2,940)$ in those without HPV vaccination, indicating a significant decrease in vaccinated women $(p=0.03)$. ASC-US cases were referred to HPV DNA tests. In addition, the rates of high grade squamous intraepithelial lesion (HSIL) or worse were $0.30 \%(1 / 332)$ in women with HPV vaccination and $0.82 \%(24 / 2,940)$ in those without HPV vaccination, showing the marginal decrease in women who were vaccinated $(p=0.3)$. Thus, this study indicates that HPV vaccination is associated with a reduction in the incidence of cervical abnormalities, suggesting a need for scientific discussion of reinstatement of proactive recommendation for HPV vaccine in Japan.

Keywords: ASC-US; cervix; cytology; HPV vaccine; uterine cervical cancer screening

Tohoku J. Exp. Med., 2016 October, 240 (2), 147-151. C 2016 Tohoku University Medical Press

\section{Introduction}

Use of human papillomavirus (HPV) vaccine started in Japan in 2009 and governmental support for vaccination began in November 2010 for girls aged 12-16 years. Since April 2013, the bivalent and quadrivalent vaccines (Cervarix and Gardasil) have been included in the national immunization program. However, due to potential adverse effects of HPV vaccination, such as chronic pain, the proactive recommendation for vaccination was suspended in June 2013 (Morimoto et al. 2014; Ueda et al. 2015; Hanley et al. 2015). The Vaccine Adverse Review Committee of the Japanese Ministry of Health investigated adverse events in about 8,898,000 injections from 2010 to 2013. Among 2,475 adverse events, there were $617(6.9 / 100,000)$ severe cases and $176(2.0 / 100,000)$ cases with chronic pain. Adverse events suspected to be associated with HPV vaccine were anaphylaxis $(1 / 960,000)$, Guillain-Barré syndrome $(1 / 4,300,000)$, acute disseminated encephalomyelitis $(1 / 4,300,000)$, and complex regional pain syndrome $(1 / 8,600,000)$. There were $2,584(29 / 100,000)$ adverse events in about 8,900,000 injections from 2010 to 2014 . The follow-up on 1,739 adverse events among 2,584 adverse events revealed that $1,550 / 1,739$ (89.1\%) cases had recovered and 186/1,739 (10.7\%) cases were not recovered in 2015. The adverse events of chronic pain may represent functional disorders, rather than organic disorders. Regardless of the cause, establishing a system for treatment is important for patients with an adverse event after injection of HPV vaccine.

Received March 25, 2016; revised and accepted September 26, 2016. Published online October 15, 2016; doi: 10.1620/tjem.240.147. Correspondence: Nobuyoshi Ozawa, Ozawa Women’s General Clinic, 192 Aramachi, Wakabayashi-ku, Sendai, Miyagi 984-0073, Japan. e-mail:nobuyosi@mva.biglobe.ne.jp 
In July 2016, a representative of an organization of people affected by HPV vaccine reported that sixty-three girls had filed lawsuits against the Japanese Government and two vaccine companies. To date, the Japanese Government has not reinstated a proactive recommendation for use of HPV vaccine. The risks of the vaccine are easily understood, whereas the merits of the vaccine are not easily understood. Therefore, we examined the short-term effects of HPV vaccine in Japan by investigating vaccination rates and the incidence of abnormal cytology.

\section{Subjects and Methods}

The subjects were women aged 20 to 24 years who underwent uterine cervical cancer screening in Miyagi Prefecture in the fiscal year (FY) 2014 (April 2014-March 2015). We asked each subject to report her history of vaccination in a questionnaire. Cervical cytology and history of vaccination were also examined using data of the Miyagi Cancer Society. Statistical analysis was performed using the $\chi^{2}$ test.

The present study and the questionnaire were discussed by the Gynecological Exam Committee of Miyagi Cancer Society and were approved by individual municipal governments. This study was approved by the ethics committee of Miyagi Cancer Society (approved number 1508).

\section{Results}

Among the 3,272 women aged 20-24 years who underwent a health check in FY2014, 332 (10.2\%) had received a HPV vaccination (Table 1). The rates of vaccination were $42.3 \%, 10.0 \%, 17.5 \%, 3.8 \%$, and $4.0 \%$ in those aged 20 , $21,22,23$, and 24 years, respectively (Table 1 ).

The effect of HPV vaccination was evaluated, based on the Bethesda System for cervical cytology (Fig. 1). The overall results of cervical cytology are summarized in Table 2. Cases of atypical squamous cells of undetermined significance (ASC-US) or worse include all categories, except for negative for intraepithelial lesion or malignancy (NILM). ASC-US cases were subjected to HPV DNA tests and further colposcopic examinations, whereas there was no need to do further examinations for cases of NILM. The rates of ASC-US or worse were $2.41 \%(8 / 332)$ in women with HPV vaccination and 5.03\% $(148 / 2,940)$ in cases without HPV vaccination (Table 3). These results indicate that vaccination resulted in a significant decrease in the rate of ASC-US or worse $(\mathrm{p}=0.03)$. The extent of the reduction was $52.1 \%$.

Table 4 summarizes the cases of high grade squamous intraepithelial lesion (HSIL) or worse and cases of low grade squamous intraepithelial lesion (LSIL) or better. Cases of HSIL or worse include HSIL, squamous cell carcinoma (SCC), atypical glandular cells (AGC), adenocarcinoma in situ (AIS), adenocarcinoma, and other malignancy, while cases of LSIL or better include all categories, except for HSIL, SCC, AGC, AIS, adenocarcinoma and other malignancy (see Fig. 1). The cases of HSIL or worse were likely to be treated after colposcope. In contrast, most cases of LSIL or better were not necessarily treated. The rates of HSIL or worse were $0.30 \%(1 / 332)$ in vaccinated women and $0.82 \%(24 / 2,940)$ in those without HPV vaccination (Table 5). The rate of HSIL or worse was lower in vaccinated subjects, although the degree of the decline was not statistically significant $(p=0.3)$. The extent of the reduction was $63.4 \%$.

\section{Discussion}

The vaccination rate of women aged 20 years who underwent uterine cervical cancer screening was $42.3 \%$ in FY2014. It is likely that women who undergo vaccination will receive uterine cervical cancer screening in the coming years. In addition, the beneficial effects of vaccination may become more apparent over time. In this study, we compared the results of cervical cytology in women aged 20 to 24 years with and those without vaccination. The rate of ASC-US or worse was significantly lower and the rate of HSIL or worse tended to be lower in women who had undergone HPV vaccination.

Several clinical trials have shown the effects of quadrivalent HPV vaccine (Villa et al. 2007) and bivalent HPV vaccine (Paavonen et al. 2009). In Australia, Crowe et al. (2014) showed a $43 \%$ decrease in the rate of atypical cells and a $62 \%$ decrease of HSIL in women aged 22-25 years

Table 1. Rates of vaccination for HPV among the women who underwent cervical cancer screening in FY2014.

\begin{tabular}{|c|c|c|c|c|c|c|}
\hline & $\mathbf{2 0}$ y.o. & $\mathbf{2 1} \mathbf{y . 0 .}$ & $\mathbf{2 2} \mathbf{y . 0}$ & $\mathbf{2 3} \mathbf{y . 0 .}$ & $\mathbf{2 4} \mathbf{y . 0 .}$ & $\begin{array}{c}\text { Total } \\
\text { cases }\end{array}$ \\
\hline $\begin{array}{c}\text { HPV } \\
\text { vaccination (+) }\end{array}$ & 112 & 78 & 75 & 29 & 38 & 332 \\
\hline $\begin{array}{c}\text { HPV } \\
\text { vaccination (-) }\end{array}$ & 153 & 720 & 428 & 728 & 911 & 2,940 \\
\hline Total cases & 265 & 798 & 503 & 757 & 949 & 3,272 \\
\hline Rate & $42.3 \%$ & $10.0 \%$ & $17.5 \%$ & $3.8 \%$ & $4.0 \%$ & $10.2 \%$ \\
\hline
\end{tabular}




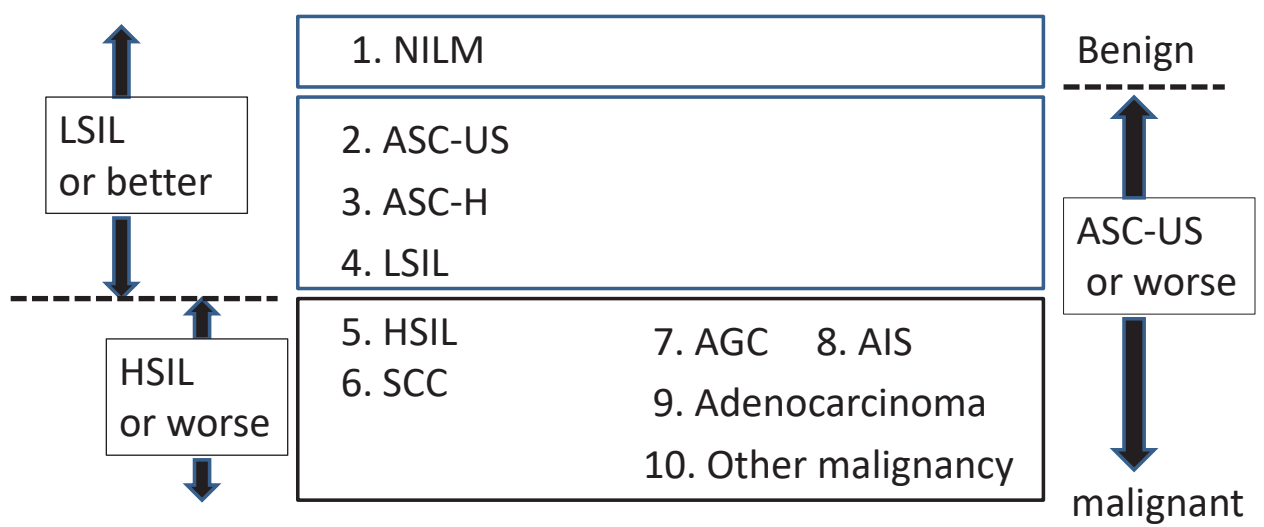

Fig. 1. The Bethesda System for reporting cervical cytology.

The morphologic findings are categorized, as shown below.

NILM, negative for intraepithelial lesion or malignancy; ASC-US, atypical squamous cells of undetermined significance; ASC-H, atypical squamous cells, cannot exclude HSIL; LSIL, low grade squamous intraepithelial lesion; HSIL, high grade squamous intraepithelial lesion; SCC, squamous cell carcinoma; AGC, atypical glandular cells; AIS, adenocarcinoma in situ.

Table 2. Cases of ASC-US or worse and NILM among the women with and without HPV vaccination.

\begin{tabular}{|c|c|c|c|}
\hline & ASC-US or worse & NILM & Total cases \\
\hline HPV vaccination (+) & 8 & 324 & 332 \\
\hline HPV vaccination (-) & 148 & 2,792 & 2,940 \\
\hline Total cases & 156 & 3,116 & 3,272 \\
\hline
\end{tabular}

ASC-US, atypical squamous cells of undetermined significance; NILM, negative for intraepithelial lesion or malignancy.

ASC-US or worse includes all categories except for NILM.

Table 3. Rates of ASC-US or worse among the women with and without HPV vaccination.

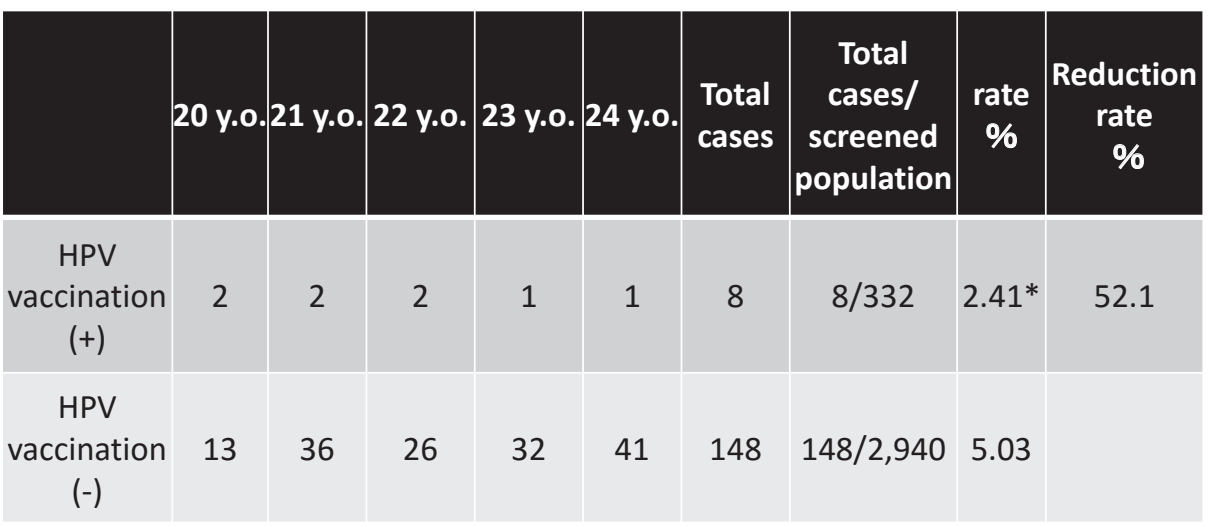

*P: 0.03 .

ASC-US, atypical squamous cells of undetermined significance.

with three vaccinations (after a lapse of 7 years). A report in Denmark (Baldur-Felskov et al. 2014) on uterine cervical cancer screening for women aged 20-21 years showed that the rates of atypical cells, cervical intraepithelial neoplasia (CIN) 2 and CIN3 decreased by about $60 \%, 73 \%$ and $80 \%$, respectively. Likewise, in Scotland, Pollock et al. (2014) showed that the rate of CIN3 decreased by about $55 \%$ after vaccination.

The subjects in Miyagi Prefecture in Japan were exam- ined only a few years after the start of HPV vaccination. We thus found that the rate of ASC-US or worse was significantly lower in vaccinated women and the rate of HSIL or worse was marginally lower in those who were vaccinated. These data are consistent with the findings in Australia, Demark and Scotland. However, in Japan, due to the potential adverse effects, such as chronic pain, the recommendation for HPV vaccination has been suspended since 2013.

Only five years have passed since commencement of 
Table 4. Cases of HSIL or worse and LSIL or better among the women with and without HPV vaccination.

\begin{tabular}{|c|c|c|c|}
\hline & HSIL or worse* & LSIL or better** & Total cases \\
\hline HPV vaccination (+) & 1 & 331 & 332 \\
\hline HPV vaccination (-) & 24 & 2,916 & 2,940 \\
\hline Total cases & 25 & 3,247 & 3,272 \\
\hline
\end{tabular}

HSIL or worse includes HSIL, squamous cell carcinoma (SCC), adenocarcinoma in situ (AIS), adenocarcinoma and other malignancy. LSIL or better includes negative for intraepithelial lesion or malignancy (NILM), ASC-US, and atypical squamous cells, cannot exclude HSIL (ASC-H).

HSIL, high grade squamous intraepithelial lesion; LSIL, low grade squamous intraepithelial lesion.

Table 5. Rates of HSIL or worse among the women with and without HPV vaccination.

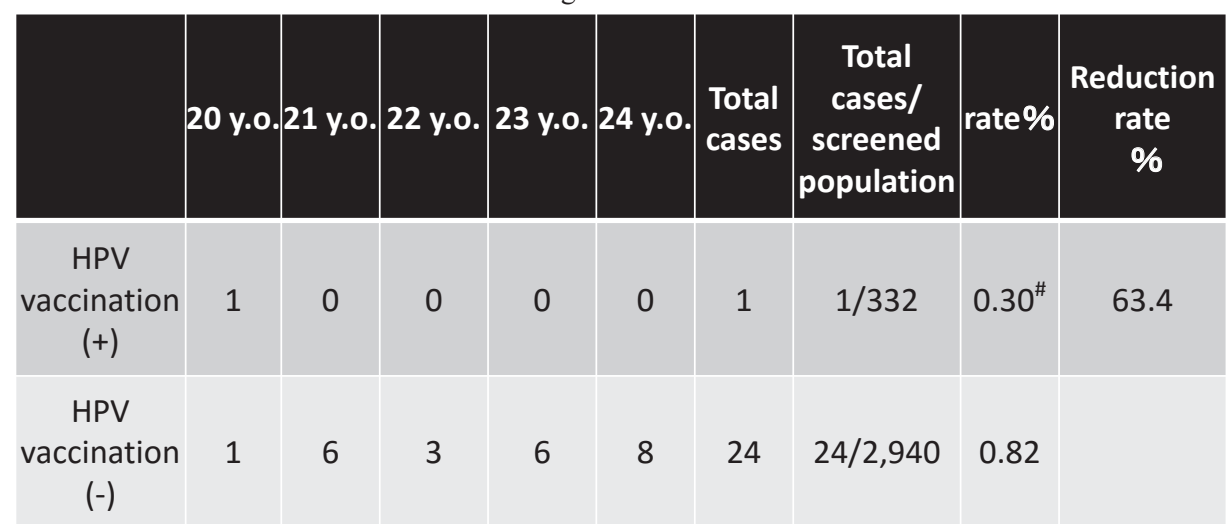

\#P: 0.3 .

HPV vaccination in Japan. The incidence of abnormal cytology found in uterine cervical cancer screening showed a noticeable difference between women with and those without a history of HPV vaccination. HPV vaccination is associated with a significant reduction in the incidence of ASC-US or worse. Therefore, further follow-up studies of HPV vaccination and cervical abnormalities are required in Japan. There is also a need to establish a system for treatment of patients with adverse events after injection of HPV vaccine.

\section{Acknowledgments}

We would like to express our deep gratitude to Mr. Masaaki Otomo, the staff members of the Miyagi Cancer Society, and physicians of the Gynecological Exam Committee for their assistance with data collection and analysis.

\section{Conflict of Interest}

The authors declare no conflict of interest.

\section{References}

Baldur-Felskov, B., Dehlendorff, C., Munk, C. \& Kjaer, S.K. (2014) Early impact of human papillomavirus vaccination on cervical neoplasia: nationwide follow-up of young Danish women. J. Natl. Cancer Inst., 106, djt460.

Crowe, E., Pandeya, N., Brotherton, J.M., Dobson, A.J., Kisely, S., Lambert, S.B. \& Whiteman, D.C. (2014) Effectiveness of quadrivalent human papillomavirus vaccine for the prevention of cervical abnormalities: case-control study nested within a population based screening programme in Australia. $B M J$, 348, g1458.

Hanley, S.J., Yoshioka, E., Ito, Y. \& Kishi, R. (2015) HPV vaccination crisis in Japan. Lancet, 385, 2571.

Morimoto, A., Ueda, Y., Egawa-Takata, T., Yagi, A., Terai, Y., Ohmichi, M., Ichimura, T., Sumi, T., Murata, H., Kanzaki, H., Nakai, H., Mandai, M., Yoshino, K., Fujita, M., Kimura, T., et al. (2014) Effect on HPV vaccination in Japan resulting from news report of adverse events and suspension of governmental recommendation for HPV vaccination. Jpn. Soc. Clin. Oncol., 20, 549-555.

Paavonen, J., Naud, P., Salmeron, J., Wheeler, C.M., Chow, S.N., Apter, D., Kitchener, H., Castellsague, X., Teixeira, J.C., Skinner, S.R., Hedrick, J., Jaisamran, U., Limson, G., Garland, S., Szarewski, A., et al. (2009) Efficacy of human papillomavirus (HPV)-16/18 AS04-adjuvanted vaccine against cervical infection and precancer caused by oncogenic HPV types (PATRICIA): final analysis of a double-blind, randomised study in young women. Lancet, 374, 301-314.

Pollock, K.G., Kavanagh, K., Potts, A., Love, J., Cuschieri, K., Cubie, H., Robertson, C., Cruickshank, M., Palmer, T.J., Nicoll, S. \& Donaghy, M. (2014) Reduction of low- and highgrade cervical abnormalities associated with high uptake of the HPV bivalent vaccine in Scotland. Br. J. Cancer, 111, 1824-1830.

Ueda, Y., Enomoto, T., Sekine, M., Egawa-Tanaka, T., Morimoto, A. \& Kimura, T. (2015) Japan's failure to vaccinate girls against human papillomavirus. Am. J. Obstet. Gynecol., 212, 405-406.

Villa, L.L., Kjaer, K., Paavonen, J., Lehtinen, M., Muñoz, N., 
Sigurdsson, K., Hermandez-Avila, M., Skjeldestad, F.E., Thoresen, S., García, P., Majewski, S., Dillner, J., Olsson, S.E., Tay, E.H., Bosch, F.X., et al. (2007) Quadrivalent vaccine against human papillomavirus to prevent high-grade cervical lesions. N. Engl. J. Med., 356, 1915-1927. 